\title{
Targeting JAK/STAT pathway in Takayasu's arteritis
}

P. Régnier ${ }^{1,2}$, A. Le Joncour ${ }^{3}$, A. Maciejewski-Duval ${ }^{1,2}$, AC. Desbois ${ }^{1,2,3}$, C. Comarmond ${ }^{1,2,}$ ${ }^{3}$, M. Rosenzwajg ${ }^{1,2}$, D. Klatzmann ${ }^{1,2}$, P. Cacoub ${ }^{1,2,3}$ and D. Saadoun ${ }^{1,2,3}$

${ }^{1}$ Sorbonne Universités, UPMC Université Paris 6, INSERM, UMR S 959, ImmunologyImmunopathology- Immunotherapy (I3); F-75005, Paris, France;

${ }^{2}$ Biotherapy (CIC-BTi) and Inflammation-Immunopathology-Biotherapy Department (DHU i2B), Hôpital de la Pitié-Salpêtrière, AP-HP, F-75651, Paris, France;

${ }^{3}$ AP-HP, Groupe Hospitalier Pitié-Salpêtrière, Department of Internal Medicine and Clinical Immunology, F-75013, Paris, France, Centre national de références Maladies Autoimmunes et systémiques rares et Maladies Autoinflammatoires rares 


\section{$\underline{\text { Abstract }}$}

\section{BACKGROUND}

Takayasu's arteritis (TAK) is a large vessel vasculitis with important infiltration of proinflammatory T cells in the aorta and its main branches, but its etiology is still unknown. Here, we explore the critical involvement of JAK/STAT signaling pathway in proinflammatory $\mathrm{T}$ cells differentiation and disease progress of TAK patients.

\section{METHODS}

We analyzed transcriptome and interferon gene signature of FACS-sorted CD4+ and CD8+ T cells from healthy donors (HD) and TAK. Then, we tested, in vitro and in vivo, the effects of JAK inhibitors (JAKinibs) in TAK patients.

\section{RESULTS}

Transcriptome analysis showed 248 significantly dysregulated genes for CD4+ samples and 432 for CD8+ samples between healthy donors (HD) and Takayasu (TAK) patients. Among dysregulated genes, a great enrichment for pathways linked to interferons (especially type I interferons), JAK/STAT signaling and cytokines/chemokines-related signaling was noticed in TAK. We confirmed by qPCR the upregulation of the interferon gene signature (ISG) signature in TAK. JAKinibs induced both in vitro and in vivo a marked reduction of CD25 expression by CD4+ and CD8+ T cells, a significant decrease of CD4+ IFN $\gamma+$ Th1 cells and of CD4+ IL-17A+ Th17 cells and an increase of CD4+ FoxP3+ CD25+ Tregs cells in TAK patients. JAKinibs also allowed decreased $\mathrm{C}$ reactive protein level and steroid dose reduction.

\section{CONCLUSIONS}

JAK/STAT signaling pathway is critical in the pathogenesis of TAK and JAKinibs may be very promising in the treatment of TAK. 


\section{Introduction}

Takayasu's arteritis (TAK) is a large vessel vasculitis (LVV) preferentially affecting the aorta and its main branches ${ }^{1}$. Vessel inflammation leads to wall thickening, fibrosis, stenosis, and, in some, complete occlusion of the artery. Although TAK has a worldwide distribution, the disease is known to be more common in young women mostly in the second or the third decade of life. Patients with TAK have a high morbidity rate, 50\% will relapse and experience a vascular complication within 10 years from diagnosis ${ }^{2,3}$.

There are several lines of evidence showing that TAK inflammation is mediated by T cells and macrophages, predominantly in the adventitial and medial layers ${ }^{4,5}$. CD4 T cells activation is driven by Th1 and Th17 cells in the peripheral blood and inflamed tissues $5,6,7,8$, ${ }^{24}$. Neovessels and adventitial vasa vasorum are the main sites where circulating leucocytes are recruited into the vascular bed ${ }^{8}$. Molecular mechanisms underlying the differentiation of pro-inflammatory $\mathrm{T}$ cells in TAK patients are essentially unknown.

Currently, TAK patients are principally treated with aspecific corticosteroids ${ }^{1}$, which are associated with potential side effects, especially when used for a long-time course. To develop more efficient treatments against this persistent and deleterious inflammation in patients, physicians need a deeper understanding of the disease and its underlying mechanisms.

One of these novel ways could be the JAK/STAT signaling cascade, which is a central biological pathway aiming at transferring ligands/receptors signals from the extracellular medium to the nucleus, finally leading to the transcription of numerous downstream genes. This pathway is involved in many critical immune functions, such as cytokines, chemokines and interferons signaling. By now, there are several novel JAK inhibitors (JAKinibs) that can inhibit JAK kinases function, allowing to dampen the downstream signaling. Different first generation JAKinibs exist and differ by their specificities: either JAK1 and JAK2 for Ruxolitinib or Baricitinib, or JAK1 and JAK3 for Tofacitinib. They decrease the global cytokine response of the cells ${ }^{5,9,10}$ and reduce both response and secretion of mainly proinflammatory cytokines by $\mathrm{T}$ cells, such as IFN $\gamma$ or IL-6. They have shown promising results in giant cell arteritis (GCA), another large vessel vasculitis ${ }^{9,11,12}$.

Here, we provided a rationale for the use of JAKinib treatments in TAK. Using transcriptomics, we showed that JAK/STAT-, interferons- and cytokines/chemokines-related genes and biological pathways are highly upregulated in TAK patients in both CD4+ and CD8+ FACS-sorted T cells. We also showed that both in vitro and in vivo, the use of JAKinibs decreased secretion of pro-inflammatory cytokines, reduced Th1 and Th17 polarization and restored the balance between Teffs and Tregs. JAKinibs were also efficient to control TAK inflammation and spare steroids in 3 TAK patients. Thus, targeting JAK/STAT signaling could represent a new therapeutic avenue in the immunomodulation in TAK patients as they act upstream of key proteins regulating inflammation events in large vessel vasculitis. 


\section{Methods}

\section{Study population}

The study population consisted of 25 consecutive TAK patients with median age of 37.6 years (IQR [27.1;50]) fulfilling the American College of Rheumatology criteria for the classification of Takayasu's arteritis ${ }^{13}$. Clinical characteristics of TAK patients are indicated in Table 1. Blood samples from 37 sex and age-matched healthy donors (HD) were obtained from the Établissement Français du Sang facility (Hôpital de la Pitié-Salpêtrière, Paris) and used as controls. The study was approved by our institutional ethics review board and was performed according to the Helsinki declaration. Three patients with active Takayasu's arteritis were treated with JAK inhibitors (JAKinib). Their medical history, treatments and outcome were collected from medical charts and are presented in the Supplementary Table 1.

\section{T cells sorting, culture and flow cytometry analysis}

PBMCs for either healthy donors (HD) or Takayasu patients (TAK) were thawed and either used unsorted (for flow cytometry analysis of whole PBMCs, see below) or FACS-sorted (CD3+ CD4+ and CD3+ CD8+ T cells) and put in culture in 48 wells plate at 1 million cells per mL using R10 culture medium (RPMI supplemented with SAB, L-glutamine and Penicillin/Streptomycin) during 5 days, concomitantly with either PBS, Tofacitinib or Ruxolinitib at the concentration of $1 \mu \mathrm{M}$. At the end of the culture, supernatants were removed and frozen, and remaining cells were washed and antibody-stained for extracellular and/or intracellular markers according to the manufacturer's instructions, then analyzed by flow cytometry. The same antibody staining protocols for flow cytometry analysis were also applied on unsorted thawed whole PBMCs. For other depicted experiments such as RT-qPCR targeting specific genes, freshly FACS-sorted CD4+ and CD8+ T cells were directly put into Trizol to protect nucleic acids from degradation (see below). Nevertheless, a special protocol was applied for the flow cytometry analysis of pSTAT5A in whole PBMCs: IL-2 (180UI per sample) stimulation of cells for $15 \mathrm{~min}$ at $37^{\circ} \mathrm{C}$ into $\mathrm{R} 10$ was performed. Then, cells were immediately fixed using PFA (final concentration of 2\% PFA) for 30min in the dark at room temperature. Next, cells were centrifuged, supernatants were removed, and cells were resuspended in $4^{\circ} \mathrm{C}$ cold absolute methanol for $30 \mathrm{~min}$ at $4^{\circ} \mathrm{C}$. Finally, cells were washed in PBS FCS $10 \%$ and standard antibody staining was performed during $45 \mathrm{~min}$ at room temperature in the dark.

\section{Type I interferon gene activity evaluation}

We extracted from several previous publications a gene signature to evaluate the global type I interferon activity in our transcriptomic samples ${ }^{14,15,16,17,18}$. This signature, called here ISG for Interferon Signature Genes, is defined by the 8 following type I Interferon Response Genes (IRGs): LY6E, HERC5, IFI44L, ISG15, MX1, MX2, EPSTI1 and RSAD2. To quantify such signature activity, we used the two following different methods. First, we looked for this signature at the transcriptome level (see below) using a specialized R package called GSVA developed by Hänzelmann et al. ${ }^{19}$ that allows to compute an enrichment score for a given gene signature for each patient involved in the analysis. Then, differential analysis of the presented groups was performed using the standard limma $\mathrm{R}$ package as advised by the authors to assess for significance. Then, we confirmed such data by performing RT-qPCR with the previously depicted ISG signature on FACS-sorted CD4+ and CD8+ T cells coming 
from patients PBMCs and previously pooled together and frozen into Trizol. To perform actual RT-qPCR we used SuperScript ${ }^{\mathrm{TM}}$ VILO $^{\mathrm{TM}}$ cDNA Synthesis Kit (Invitrogen ${ }^{\mathrm{TM}}$ ) according to manufacturer's instructions. After $\mathrm{Ct}$ acquisition, individual gene data were normalized to housekeeping gene GAPDH expression. Then, each gene expression (for every sample and group) was individually normalized to the mean of the HD group, in order to make results relative to the average individual gene expression of the HD group.

\section{Transcriptomic data of CD4+ and CD8+ T cells}

CD3+ T cells were isolated from PBMCs of active Takayasu (TAK) $(n=41)$ patients or healthy donors $(\mathrm{HD})(\mathrm{n}=47)$ by negative isolation using DYNABEADS $\AA$ untouched $^{\mathrm{TM}}$ Human T Cells Kit (Thermofisher Scientific) according the manufacturer's instructions. CD4 positive cells were then isolated from previous sorted $\mathrm{T}$ cells by positive selection using DYNABEADS ${ }^{\circledR}$ CD4 positive isolation kit according the manufacturer's instructions. Total RNA from CD4 positive cells was extracted using the NucleoSpin ${ }^{\circledR}$ RNA kit (MachereyNagel), according to the manufacturer's instructions. Total RNA was quantified by a NanoDrop ND-1000 spectrophotometer. Samples with RNA concentration $<20 \mathrm{ng} / \mu \mathrm{L}$ were excluded. For quality control, RNA dilution was performed using Agilent RNA 6000 Nano Kit and $1 \mu \mathrm{L}$ of the sample was run on the Nano chip using an Agilent 2100 electrophoresis bioanalyzer. The quality of total RNA was assessed using the profile of the electropherogram and the RNA integrity number (RIN) was calculated. The whole samples showed RINs between 7.3 and 9.3. For Illumina Beadarrays, cRNA samples were prepared using Illumina TotalPre-96 RNA Amp kit (LifeTechnologies) and hybridized to Illumina Human HT-12 v4 Beadarrays. Then, raw IDAT files were processed using illuminaio $\mathrm{R}$ package and concatenated into a single text file summarizing all patients and genes. Data were further background-corrected using limma $\mathrm{R}$ package and inter-chip batch effects were also removed for each patient group using ComBat method from sva R package. After exclusion of lowquality samples, normalization and batch correction, the following samples numbers remained and were analyzed: $37 \mathrm{CD} 4+\mathrm{T}$ cells and $34 \mathrm{CD} 8+\mathrm{T}$ cells from $\mathrm{HD}$, and $25 \mathrm{CD} 4+\mathrm{T}$ cells and $14 \mathrm{CD} 8+\mathrm{T}$ cells from active TAK patients.

\section{Construction of gene networks}

Gene networks were constructed using the igraph and GSVA R packages coupled with human protein interactome from STRING database using a (high) confidence cut-off score of 950 out of 1000 and biological pathways extracted from 5 different well-known annotated databases: GeneOntology, Reactome, KEGG, Panther and WikiPathways. This allowed us to connect genes coming from 1 or more selected pathways according to their interaction at the protein level. Then, actual networks were generated directly in $\mathrm{R}$ using the previously depicted data and exported to SVF format in order to be edited in Adobe Illustrator CC 2018 to change the nodes color to a more readable color scale : grey for low node degree (low number of connections with other nodes), black for intermediate node degree, orange for high node degree and red for very high node degree. Finally, only the nodes from the 2 last categories were eventually annotated with the gene symbol in order to ease the graph interpretation. 


\section{$\underline{\text { Results }}$}

\section{Interferon gene signature in T cells of Takayasu patients}

First, we performed direct differential transcriptome analysis between healthy donors (HD) and Takayasu (TAK) patients for both CD4+ and CD8+ T cells. We obtained 248 significantly dysregulated genes (with adjusted p-values threshold defined to 0.05) for CD4+ samples (148 enriched in HD and 100 enriched in TAK patients) and 432 for CD8+ samples (254 enriched in HD and 178 enriched in TAK patients). Among dysregulated genes, we noticed a great enrichment for genes involved in T cells activation pathways, response to cytokines/chemokines, interferons response/synthesis and immune response in both CD4+ and CD8+ T cells of TAK patients. We wanted to confirm interferon gene signature in TAK patients, and thus we evaluated the type I interferon activity in our TAK samples compared to HD, by using a global type I interferon activity gene signature (called ISG) generated from previous publications ${ }^{14,15,16,17,18}$. We plotted all the genes' normalized enrichment percentages between CD4+ (Figure 1A) or CD8+ (Figure 1B) cells of HD and TAK patients. We clearly see a great enrichment of genes composing the ISG signature (6 out of 8 genes statistically significant for both CD4+- ranging from $8.67 \%$ to $32.36 \%$ of the greatest gene enrichment - and CD8+ samples - ranging from $7.22 \%$ to $46.91 \%$ of the greatest gene enrichment) toward the TAK patients. Then, we confirmed this observation by calculating an ISG signature enrichment score using GSVA package as previously described ${ }^{19}$. This led to individual ISG enrichment scores that we were able to compare between HD and TAK patients using standard limma differential analysis $\mathrm{R}$ package. This protocol was applied on both CD4+ cells (Figure 1C) or CD8+ cells (Figure 1D) from HD and TAK patients. In both situations, we have seen a clear and significant enrichment of this ISG signature in TAK patients as compared to HD, which tends to be even greater in CD8+ samples. Furthermore, we performed RT-qPCR for the same genes on pooled FACS-sorted CD4+ and CD8+ T cells coming from either HD or TAK patients, in order to eliminate non-T cells contaminants and to establish a gene expression quantification regarding real CD3+ T cells (Figure 1E). We confirmed by this technique the upregulation of the ISG signature in TAK versus HD in T cells. Together, our results strongly demonstrate that the type I interferon-related gene expression is greatly upregulated in TAK.

\section{JAK-STAT pathway signature efficiently separates CD4+ T cells from healthy donors and Takayasu patients}

Next, we focused on CD4+ samples coming from HD or TAK patients and performed a full pathway enrichment analysis using the combination of 5 gene sets databases (Gene Ontology, KEGG, Reactome, PANTHER and WikiPathways) as our source for pathways, plus GSVA/limma combo as analysis method as previously described ${ }^{19}$. This allowed us to shift from individual genes to biological functions information, by determining which pathways are significantly enriched in our condition of interest among all the 5370 available pathways. Among the 2286 significantly enriched pathways between HD and TAK patients (with an adjusted p-value threshold defined to 0.05), 996 and 1290 were enriched in HD and in TAK patients, respectively. To reduce the complexity of the results, we first generated a network of enriched pathways linked together according to their degree of overlap, in order to better visualize the major dysregulated functions between HD and TAK patients. We noticed a significant enrichment of pathways linked to interferons (especially type I interferons), JAK/STAT signaling and cytokines/chemokines-related signaling pathways among the ones greatly enriched in TAK patients (Figure S1A). Then, we decided to focus on such biological 
functions and extracted the main corresponding pathways that we plotted together on a heatmap (all have an individual adjusted $p$-value $\leq 0.05$ and separate very efficiently HD and TAK patients) (Figure 2A). We created a network of all the genes contained in the pathways presented in the heatmap using the STRING interaction database (Figure 2B). Among the highest degree nodes, we found JAK1, JAK2, STAT1, STAT2, STAT3, CCL20, CXCL10, CXCL12 and IRF9 with more than 30 connections, and JAK3, STAT5A and STAT5B with 22 to 30 connections. These genes are mainly involved in JAK/STAT signaling pathways, either via interferons or cytokines/chemokines upstream stimuli, thus confirming the great implication of the JAK/STAT signaling pathways in the physiopathology of TAK disease in CD4+ samples versus HD. STAT5A gene mRNA was significantly upregulated in both CD4+ $(\mathrm{p} \approx 0.0011)$ and $\mathrm{CD} 8+$ samples $\left(\mathrm{p} \approx 6.85 \mathrm{e}^{-8}\right)$ of TAK versus HD. To confirm its involvement, we showed, by flow cytometry a significant increased proportion of pSTAT5A+ cells in TAK patients compared to HD (Figure 2C).

\section{JAK-STAT pathway signature efficiently separates CD8+T cells from healthy donors and Takayasu patients}

Then, we performed the same operations on CD8+ samples coming from HD and TAK patients and displayed the obtained results following the same pattern as previously: among the 2772 significantly enriched pathways between HD and TAK patients (with an adjusted pvalue threshold defined to 0.05), 756 and 2016 were enriched in HD and in TAK patients, respectively. Again, we noticed a great enrichment in TAK versus HD of pathways related to interferons- and JAK/STAT signaling pathways and response to cytokines/chemokines (Figure S1B) and focused on such biological functions. We extracted the main corresponding pathways and plotted them on a single heatmap (Figure 3A). Pathways presented in the heatmap all have an individual adjusted $p$-value $<0.05$ and separate very efficiently HD and TAK patients. The 2286 pathways significantly enriched in CD4+ TAK samples versus HD and the 2772 pathways significantly enriched in CD8+ TAK samples versus HD shared 1127 common pathways (respectively about $49.3 \%$ and $40.7 \%$ of each group pathways), indicating the proximity of the upregulated pathways in CD4+ and CD8+ T cells in TAK patients. In the Figure 3B, we created a gene network of all the genes contained in the previously depicted pathways. As previously, we also confirmed the upregulation of pSTAT5A in CD8+ samples of TAK as compared to HD (Figure 3C). Together, our results clearly show that JAK/STAT pathway is critical for the immunopathology of TAK. These data provide us with a rationale to target JAK/STAT in TAK.

\section{JAK inhibitors reduce T cells activation, Th1 and Th17 polarization and promote increase of Tregs in TAK}

Next, we aimed to study the effect of Ruxolitinib (anti-JAK1 and JAK2) on FACS-sorted T cells (either CD4+ or CD8+) coming from PBMCs of HD or TAK patients and cultured for 5 days with or without Ruxolitinib. Ruxolitinib induced a marked reduction of CD25 expression by CD4+ and CD8+ T cells (in percentage and MFI) in TAK patients (Figure 4A and Figure 4B, first line). This effect was also present for HD. Ruxolitinib treatment also induced decrease of CD4+ IFN $\gamma+$ Th1 cells and increase of CD4+ FoxP3+ CD25+ Tregs cells in TAK patients but not in HD (Figure 4A, second line). These trends were also seen in CD8+ T cells but did not reach statistical significance (Figure 4B, second line). Finally, Ruxolitinib induced a significant decrease of CD4+ IL-17A+ Th17 cells in TAK patients (Figure 4A, third line). Together, these results confirm the beneficial in vitro effect of Ruxolitinib on $\mathrm{T}$ 
cells activation/differentiation in TAK patients, by restoring the balance between effector and regulatory $\mathrm{T}$ cells.

\section{Targeting JAK-STAT pathway with JAK inhibitors improve TAK}

To confirm our previous in vitro results, we treated 3 of our TAK patients with JAK inhibitors (JAKinibs) as detailed in Figure 5 and Supplementary Table 1. Using flow cytometry (available for 2 patients), we found that treatment with JAK inhibitors decrease the CD25 expression by CD4+ T cells and increase Tregs percentages as soon as 1 month of treatment (Figure 5A, first line). We also confirmed that Th1 and Th17 cells were decreased and that the Tregs/Teffs ratio increased (Figure 5A, second line), thus confirming the reduction of CD4+ effector T cells activation/differentiation in vivo with JAKinibs. We also assessed the pSTAT5A+ cells among CD4+ T cells and confirmed a reduction of pSTAT5A expression as soon as 1 month of treatment (Figure 5B). The treatment effectively decreases systemic inflammation, as we found a reduction of $\mathrm{C}$ reactive protein (CRP) level under JAKinibs in two out of three treated TAK patients (Figure 5C, left). JAKinibs treatment also allowed steroid dose reduction in two TAK patients (Figure 5C, right). 


\section{$\underline{\text { Discussion }}$}

We identified for the first time the JAK/STAT signaling pathway as the most potent and central biological function linking major dysbalance between effector (Th1 and Th17) and regulatory T cells in TAK. Using transcriptomics analysis, we showed that JAK/STAT-, interferons- and cytokines/chemokines-related genes were highly upregulated in TAK patients in both CD4+ and CD8+ FACS-sorted T cells.

We demonstrated that interferons (and especially type I IFNs) are greatly implicated in the physiopathology of TAK, with both IFN-related gene signature and associated biological pathways upregulation in TAK using transcriptome and RT-qPCR. The ISG signature (MX1, MX2, RSAD2, LY6E, HERC5, IFI44L, EPSTI1 and ISG15) that we used only contains genes that are upregulated when an interferon stimulation is present. It allows to specifically estimate the stimulation's strength of the IFN-related signaling pathways in cells. A large body of evidence implicates type I IFNs in the development of autoimmunity: multiple rheumatologic diseases including Rheumatoid Arthritis (RA), Systemic Lupus Erythematosus (SLE), Systemic Sclerosis (SSc) and myositis are characterized by activation of type I IFN signalling ${ }^{18,20}$. Mendelian disorders associated with upregulation of type I IFN signaling also present with severe inflammation and autoimmunity ${ }^{21}$. Zhang et al. previously showed in a GCA mice model that IFN $\alpha$ and IFN $\gamma$, STAT1, STAT2 and STAT4, but also specific downstream associated genes (respectively Tbet and CXCL9 for STAT1 and ISG15 and OAS1 for STAT2) are significantly upregulated ${ }^{9}$.

Our genes network analyses allowed us to isolate among the most representative dysregulated genes in TAK (i.e. JAKs (JAK1, JAK2 and JAK3) and STATs (STAT1, STAT2, STAT3, STAT5A and STAT5B genes) the ones the that are most likely to be at the center of the network. Type I and II cytokines signal through the JAK-STAT pathway are major drivers of autoimmune and inflammatory disorders as they enhance Th1 and Th17 cell responses. Although the relative contribution of Th1 versus Th17 cells is the subject of ongoing research, both subsets have been implicated in large vessel vasculitis. Pro-inflammatory Th1 and Th17 cells are dominant infiltrates in the vascular walls, producing IFN- $\gamma$ and IL-17 to drive the systemic and vascular manifestations of TAK. Meanwhile, glucocorticoid treatment suppresses Th1 cytokines but spares Th17 cytokines in patients with TAK ${ }^{6}$. Thus, alternative therapeutic approaches for management of TAK patients are urgently needed.

We demonstrated here that JAKinibs can be used in TAK in order to dampen and eventually control overwhelming inflammatory responses in patients. Our in vitro data showed that Ruxolitinib leads to reduced Th1 and Th17 cell differentiation and increased proportion of Tregs in TAK as compared to HD. We also confirmed in vivo that JAKinibs could efficiently regulate inflammatory responses and restore $\mathrm{T}$ cell homeostasis in TAK patients. This is consistent with previous works in GCA mice showing that Tofacitinib reduced quantities of IL-17A, IFN $\gamma$ and IL-21 mRNAs in vascular lesions as compared to untreated mice ${ }^{9}$. Tofacitinib also suppressed multiple effector T cell lineages commitment (significant decrease of ROR $\gamma \mathrm{c}$, Tbet and Bcl6 mRNAs) and their subsequent cytokines secretion (IFN $\gamma$, IL-17A and IL-21 mRNAs), but also global T cells expansion, inflammation-associated microangiogenesis and hyperplasia.

Together, our findings bring a strong rationale for considering JAKinibs as a new potent therapy in TAK, used to dampen deleterious immune responses and over-activated signaling pathways. JAK/STAT signaling pathway appears as a central biological function linking 
major dysbalance between effector (Th1 and Th17) and regulatory T cells in TAK. Further investigations are warranted in order to assess their reliability and usability in TAK. 


\section{Bibliography}

1. Numano, F., Okawara, M., Inomata, H. \& Kobayashi, Y. Takayasu's arteritis. The Lancet 356, 1023-1025 (2000).

2. Comarmond, C. et al. Long-Term Outcomes and Prognostic Factors of Complications in Takayasu Arteritis: A Multicenter Study of 318 Patients. Circulation 136, 1114-1122 (2017).

3. Mirouse, A. et al. Overall survival and mortality risk factors in Takayasu's arteritis: A multicenter study of 318 patients. J. Autoimmun. 96, 35-39 (2019).

4. Weyand, C. M. \& Goronzy, J. J. Immune mechanisms in medium and large-vessel vasculitis. Nat. Rev. Rheumatol. 9, 731-740 (2013).

5. Weyand, C. M. et al. Cytokines, growth factors and proteases in medium and large vessel vasculitis. Clin. Immunol. 206, 33-41 (2019).

6. Saadoun, D. et al. Th1 and Th17 Cytokines Drive Inflammation in Takayasu Arteritis: Th1 AND Th17 CELLS in Takayasu Arteritis. Arthritis Rheumatol. 67, 1353-1360 (2015).

7. Mirault, T., Guillet, H. \& Messas, E. Immune response in Takayasu arteritis. Presse Médicale 46, e189-e196 (2017).

8. Savioli, B., Abdulahad, W. H., Brouwer, E., Kallenberg, C. G. M. \& de Souza, A. W. S. Are cytokines and chemokines suitable biomarkers for Takayasu arteritis? Autoimmun. Rev. 16, 1071-1078 (2017).

9. Zhang, H. et al. Inhibition of JAK-STAT Signaling Suppresses Pathogenic Immune Responses in Medium and Large Vessel Vasculitis. Circulation 137, 1934-1948 (2018).

10. Schwartz, D. M., Bonelli, M., Gadina, M. \& O'Shea, J. J. Type I/II cytokines, JAKs, and new strategies for treating autoimmune diseases. Nat. Rev. Rheumatol. 12, 25-36 (2016).

11. Fragoulis, G. E., McInnes, I. B. \& Siebert, S. JAK-inhibitors. New players in the field of immune-mediated diseases, beyond rheumatoid arthritis. Rheumatology 58, i43-i54 (2019).

12. Low, C. \& Conway, R. Current advances in the treatment of giant cell arteritis: the role of biologics. Ther. Adv. Musculoskelet. Dis. 11, 1759720X1982722 (2019).

13. Arend, W. P. et al. The American College of Rheumatology 1990 criteria for the classification of takayasu arteritis. Arthritis Rheum. 33, 1129-1134 (2010).

14. Raterman, H. G. et al. The interferon type I signature towards prediction of nonresponse to rituximab in rheumatoid arthritis patients. Ann. Rheum. Dis. 71, A5.1-A5 (2012).

15. Palli, E., Kravvariti, E. \& Tektonidou, M. G. Type I Interferon Signature in Primary Antiphospholipid Syndrome: Clinical and Laboratory Associations. Front. Immunol. 10, (2019).

16. Cooles, F. A. H. et al. The interferon gene signature is increased in patients with early treatment-naive rheumatoid arthritis and predicts a poorer response to initial therapy. $J$. Allergy Clin. Immunol. 141, 445-448.e4 (2018). 
17. Vosslamber, S. et al. Pharmacological induction of interferon type I activity following treatment with rituximab determines clinical response in rheumatoid arthritis. Ann. Rheum. Dis. 70, 1153-1159 (2011).

18. Higgs, B. W. et al. Patients with systemic lupus erythematosus, myositis, rheumatoid arthritis and scleroderma share activation of a common type I interferon pathway. Ann. Rheum. Dis. 70, 2029-2036 (2011).

19. Hänzelmann, S., Castelo, R. \& Guinney, J. GSVA: gene set variation analysis for microarray and RNA-Seq data. BMC Bioinformatics 14, 7 (2013).

20. Rönnblom, L. \& Eloranta, M.-L. The interferon signature in autoimmune diseases: Curr. Opin. Rheumatol. 25, 248-253 (2013).

21. Crow, Y. J. Type I interferonopathies: a novel set of inborn errors of immunity: Type I interferonopathies. Ann. N. Y. Acad. Sci. 1238, 91-98 (2011).

22. Subramanyan, R., Joy, J. \& Balakrishnan, K. G. Natural history of aortoarteritis (Takayasu's disease). Circulation 80, 429-437 (1989).

23. Chasset, F. \& Francès, C. Cutaneous Manifestations of Medium- and Large-Vessel Vasculitis. Clin. Rev. Allergy Immunol. 53, 452-468 (2017).

24. Misra, D. P., Chaurasia, S. \& Misra, R. Increased Circulating Th17 Cells, Serum IL17A, and IL-23 in Takayasu Arteritis. Autoimmune Dis. 2016, 1-8 (2016). 


\section{Figures legends}

Figure 1 - Upregulation of IFN signaling-related gene signature in TAK. (A-B) An IFN signaling-related gene signature was analyzed gene-by-gene in TAK and HD transcriptomic microarray samples of FACS-sorted CD4+ T cells (A) or CD8+ T cells (B). Normalized fold changes are relative to the absolute maximal fold change available within the full differential transcriptome analysis between HD and TAK samples. P-values were corrected using the Benjamini-Hochberg procedure. (C-D) Then, the signature was analyzed as a set of genes using GSVA R package and subsequent enrichment scores were computed for each HD and TAK sample of FACS-sorted CD4+ T cells (C) or CD8+ T cells (D). Then, standard t-tests were performed to compare the distributions of enrichment scores between HD and TAK. (E) The same gene signature was gene-by-gene tested by RT-qPCR in FACS-sorted CD8+ T cells using different PBMCs samples from TAK and HD. Normalized LogFC were computed from the raw $\mathrm{Ct}$ values using standard $\Delta \Delta \mathrm{Ct}$ transformation method and differences between HD and TAK were assessed using standard t-tests. $*$ : p-value $<0.05, * *: \mathrm{p}$-value $<0.01, * * *: \mathrm{p}$ value $<0.001, * * * *$ : p-value $<0.0001$

Figure 2 - JAK/STAT signaling pathway efficiently separates CD4+ T cells from HD and TAK. (A) Heatmap showing selected and significantly dysregulated pathways (with Benjamini-Hochberg-adjusted p-values < 0.05) between HD and TAK CD4+ T cells samples. (B) Using the genes contained in the pathways presented in (A) and the STRING database, a gene network was constructed. Nodes were colored according to their degree (number of connections with other nodes) and eventually annotated with the associated gene name. (C) Measurement by flow cytometry of pSTAT5A percentage of expression by CD4+ T cells in different HD and TAK PBMCs samples. Standard t-test was used. ** $:$ p-value $<0.01$.

Figure 3 - JAK/STAT signaling pathway efficiently separates CD8+ T cells from HD and TAK. (A) Heatmap showing selected and significantly dysregulated pathways (with Benjamini-Hochberg-adjusted p-values $<0.05$ ) between HD and TAK CD8+ T cells samples. (B) Using the genes contained in the pathways presented in (A) and the STRING database, a gene network was constructed. Nodes were colored according to their degree (number of connections with other nodes) and eventually annotated with the associated gene name. (C) Measurement by flow cytometry of pSTAT5A percentage of expression by CD8+ T cells in different HD and TAK PBMCs samples. Standard t-test was used. $* *:$ p-value $<0.01$.

Figure 4 - JAKinibs reduce global T cells activation, Th1/Th17 polarization and promote increase of Tregs in TAK. FACS-sorted CD4+ (A) and CD8+ (B) T cells from TAK and HD PBMCs were put in culture during 5 days in complete RPMI medium with or without Ruxolitinib. Then, cells were washed and surface (CD25) and intracellular (FoxP3, IFN $\gamma$ and IL-17A) markers were antibody-stained and analyzed by flow cytometry. Standard t-tests were used. $*$ : p-value $<0.05, * *$ : p-value $<0.01, * * *:$-value $<0.001$.

Figure 5 - Blocking JAK/STAT pathway with JAKinibs improve TAK. (A) Surface (CD25) and intracellular (FoxP3, IFN $\gamma$ and IL-17A) markers of PBMCs CD4+ T cells coming from in vivo untreated (M0) and treated (M1 for month 1, M6 for month 6) matched TAK patients were analyzed by flow cytometry. (B) Measurement by flow cytometry of pSTAT5A percentage of expression by CD4+ T cells in in vivo untreated and treated matched TAK patients. (C) Blood dosage in untreated or treated matched TAK patients of CRP levels (left) and concomitant corticosteroid dose administrated to patients following JAKinib treatment (right). 
Supplementary Figure 1 - Global pathways analysis between TAK and HD samples. Transcriptomes (microarray) from FACS-sorted CD4+ (A) or CD8+ (B) T cells from HD and TAK patients were separately analyzed on the pathways level. First, a collection of 5 databases of gene sets (pathways) was generated. Then, enrichment scores for each of them were computed using GSVA R package. Finally, using igraph R package, networks of the significantly dysregulated pathways were created and manually annotated. Colors of the nodes denote the direction of enrichment: blue towards HD and red towards TAK. 2 pathways are connected if they share at least $20 \%$ of their gene content. In the meantime, clusters of pathways were automatically determined: they contain very close pathways and are likely to represent a given biological function. Afterwards, clusters were manually annotated by investigating the content of each cluster. Clusters that are highlighted in green are related to cytokines, chemokines, JAK/STAT signaling pathway, inflammation or general immune cells activation/signaling. 
A

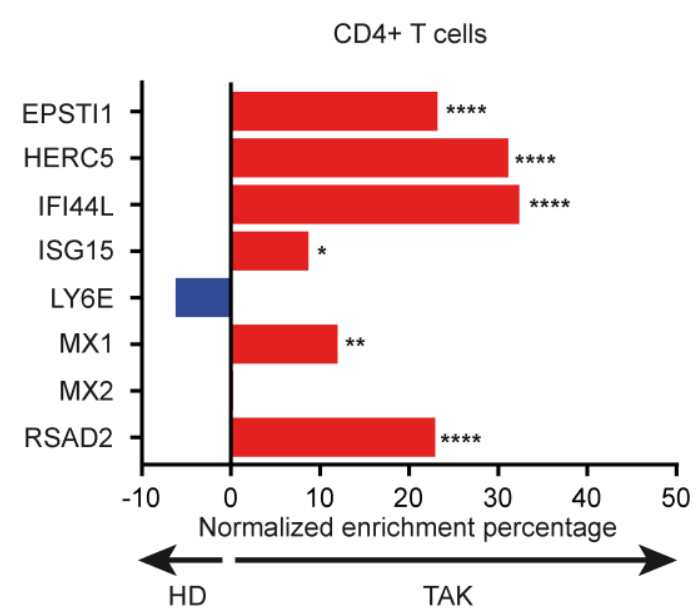

C

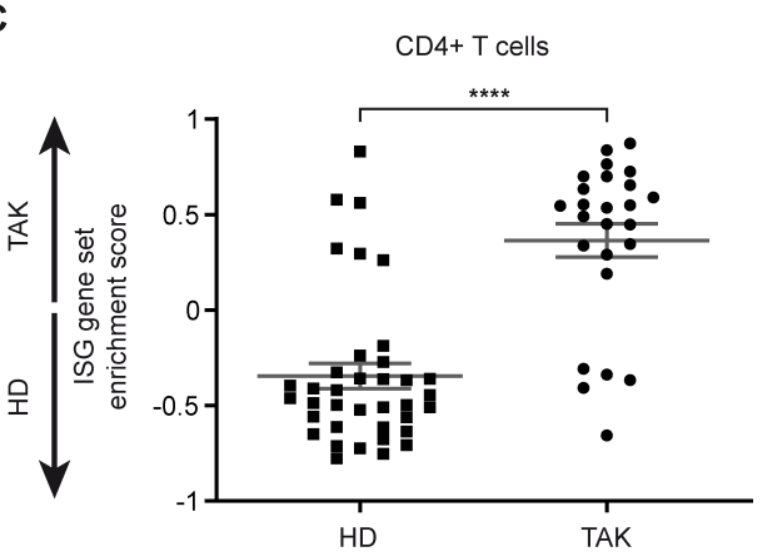

B

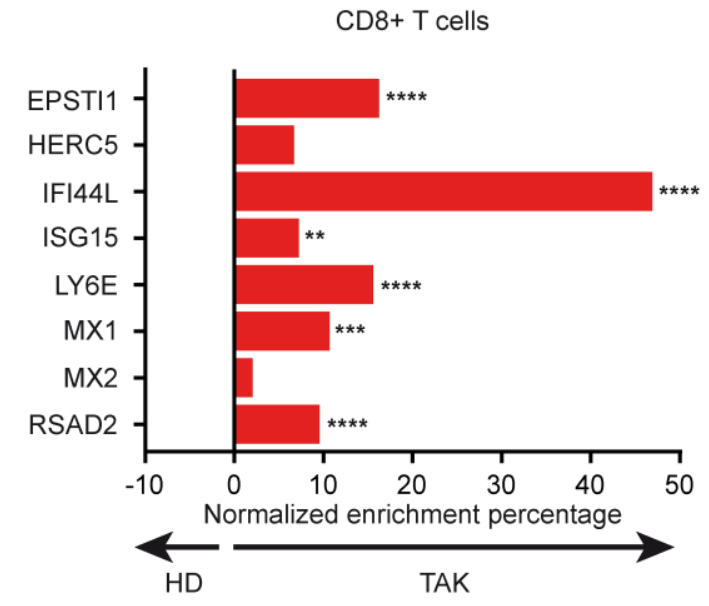

D

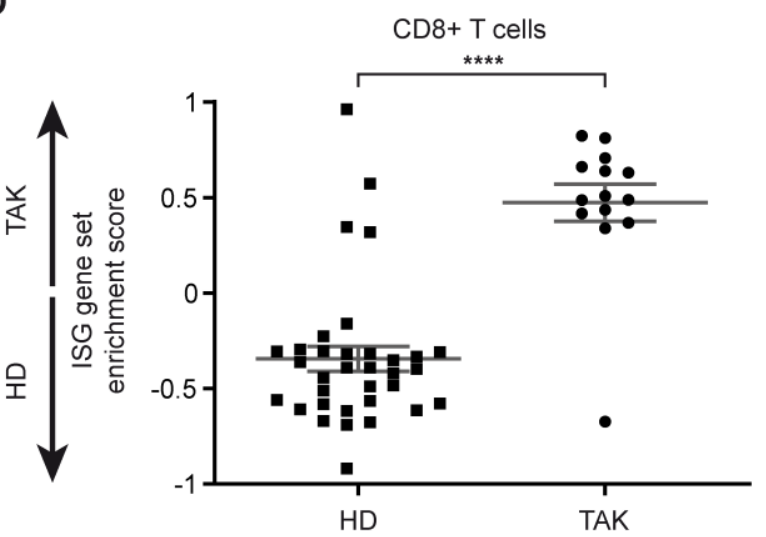

E

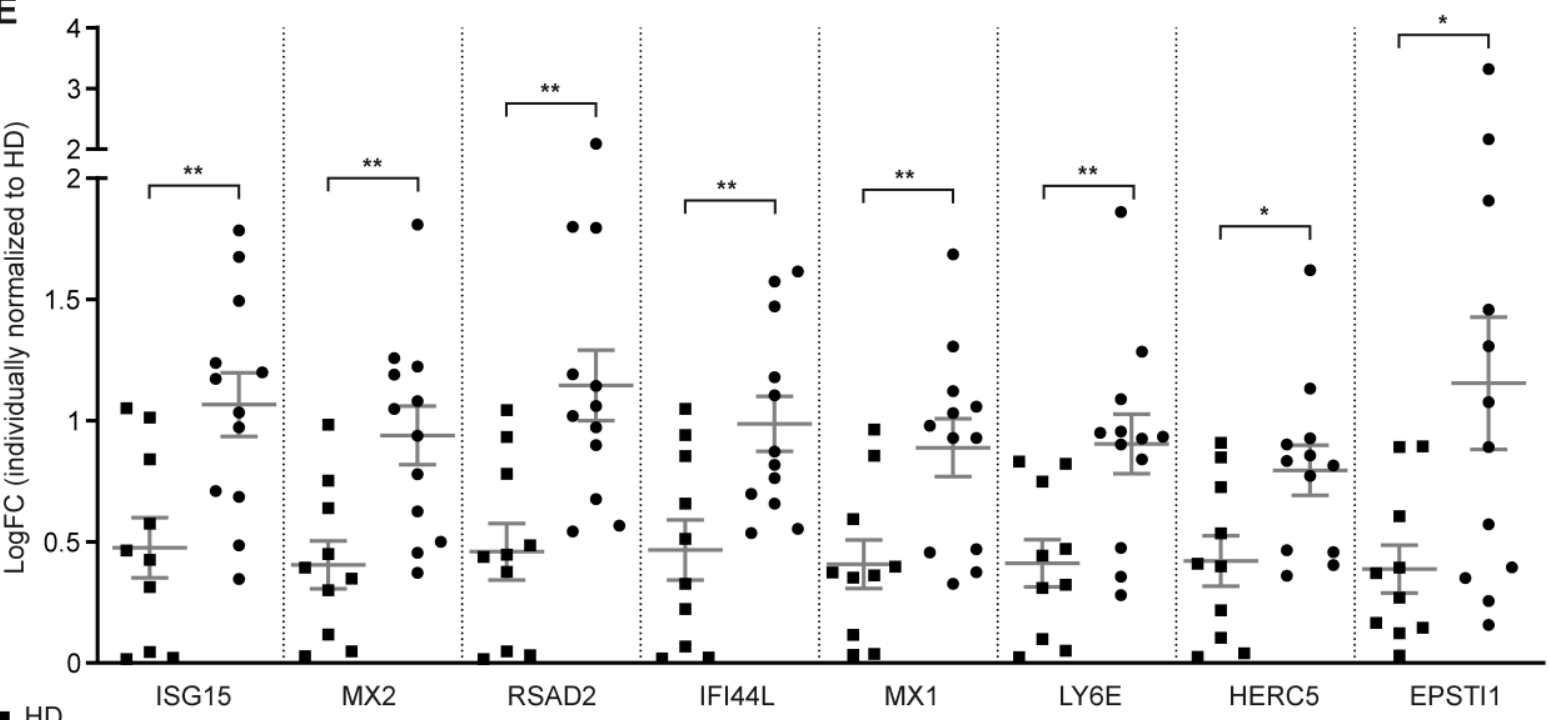

- HD

IFI44L

MX1

Figure 1 
A

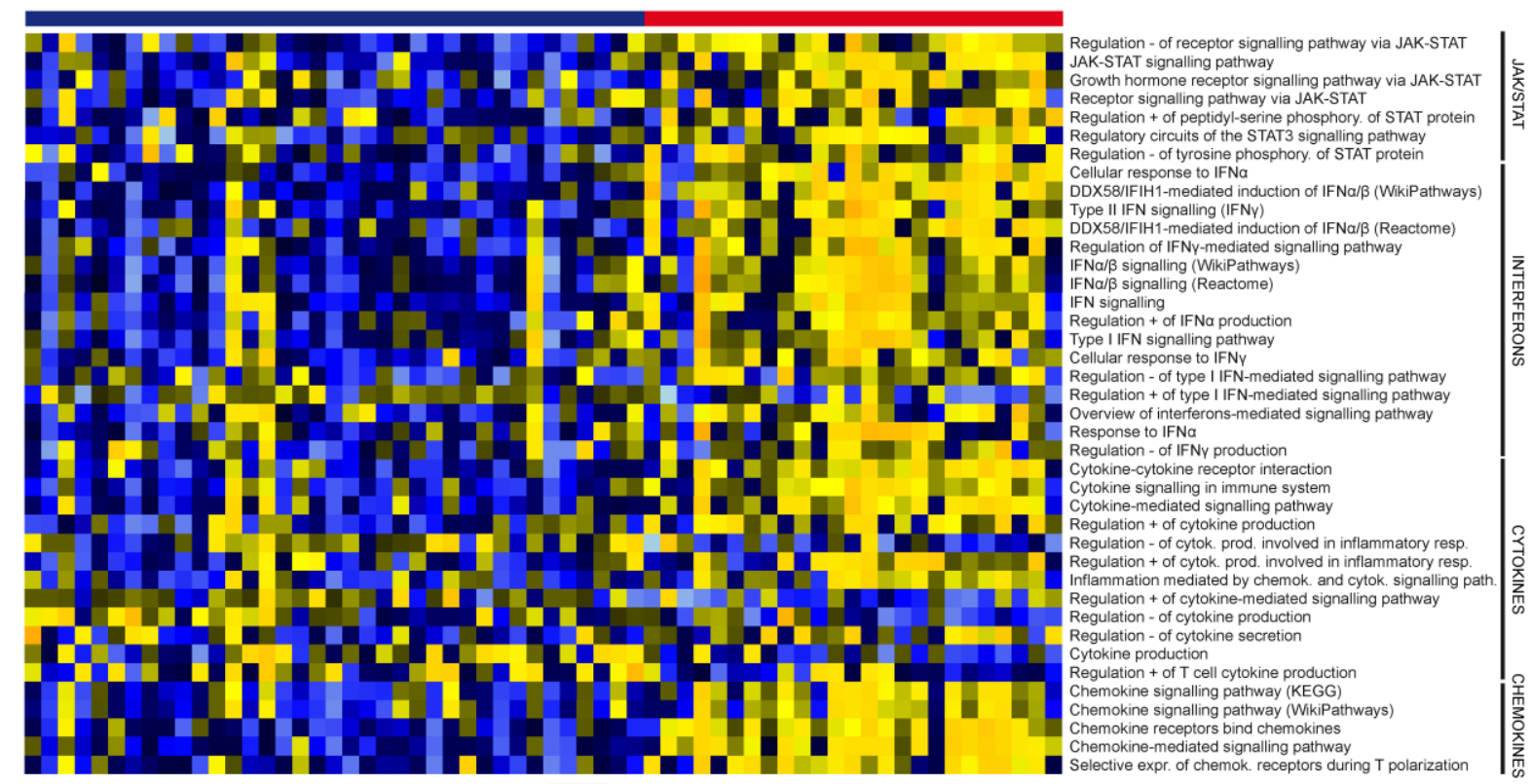

B
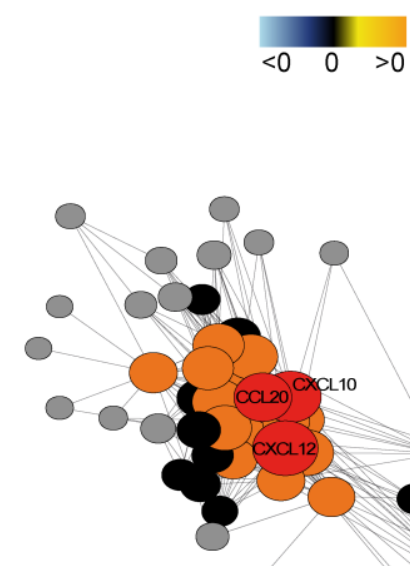

O CD4+ $T$ cells of TAK

(1)

O
C

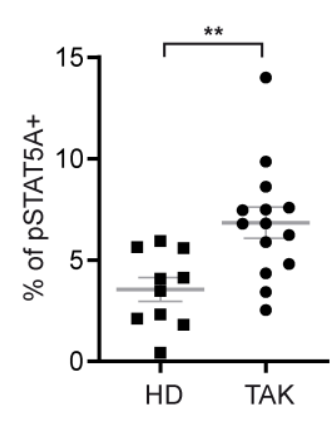

Node degree

Between 1 and 10

Between 11 and 20

Between 21 and 30

Greater than 31

Figure 2 


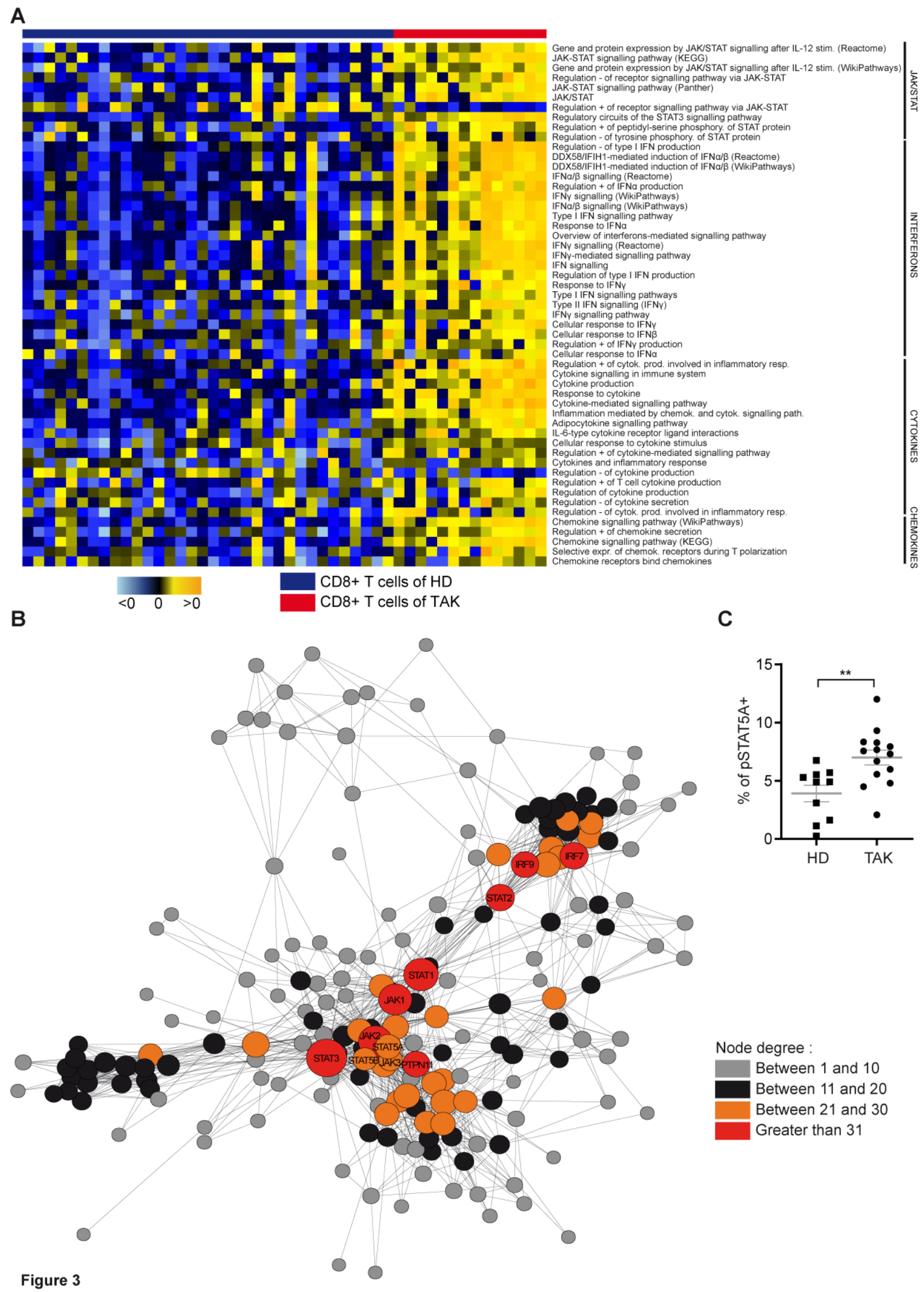


A

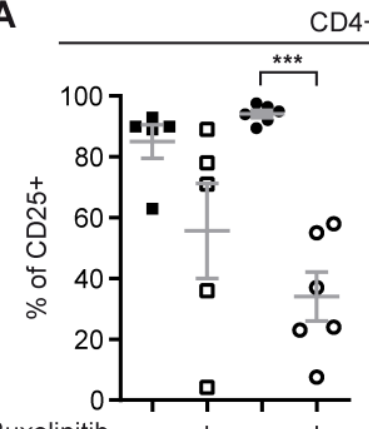

Ruxolinitib

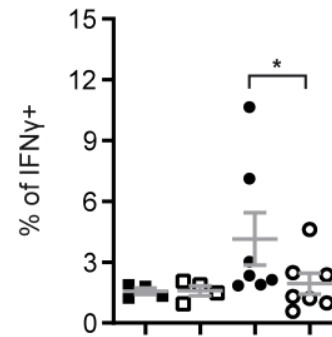

Ruxolinitib

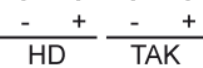
Ruxolinitib
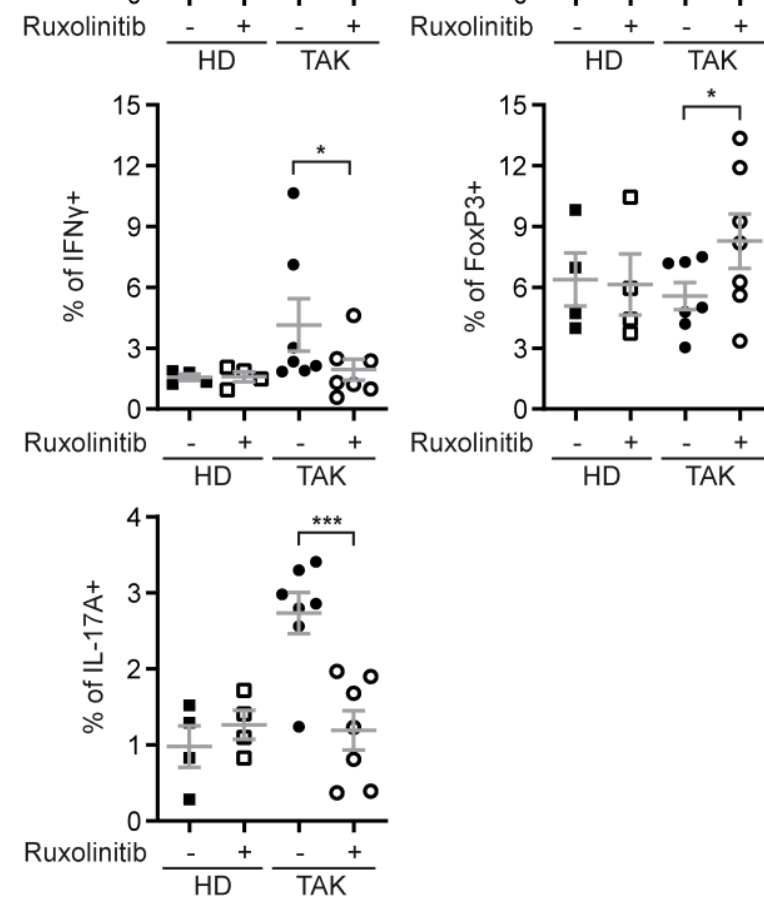

Figure 4

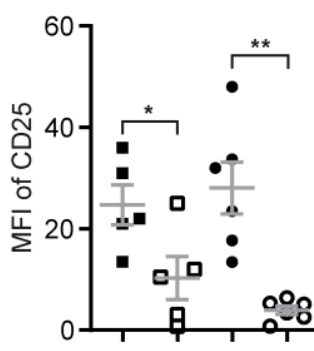

B
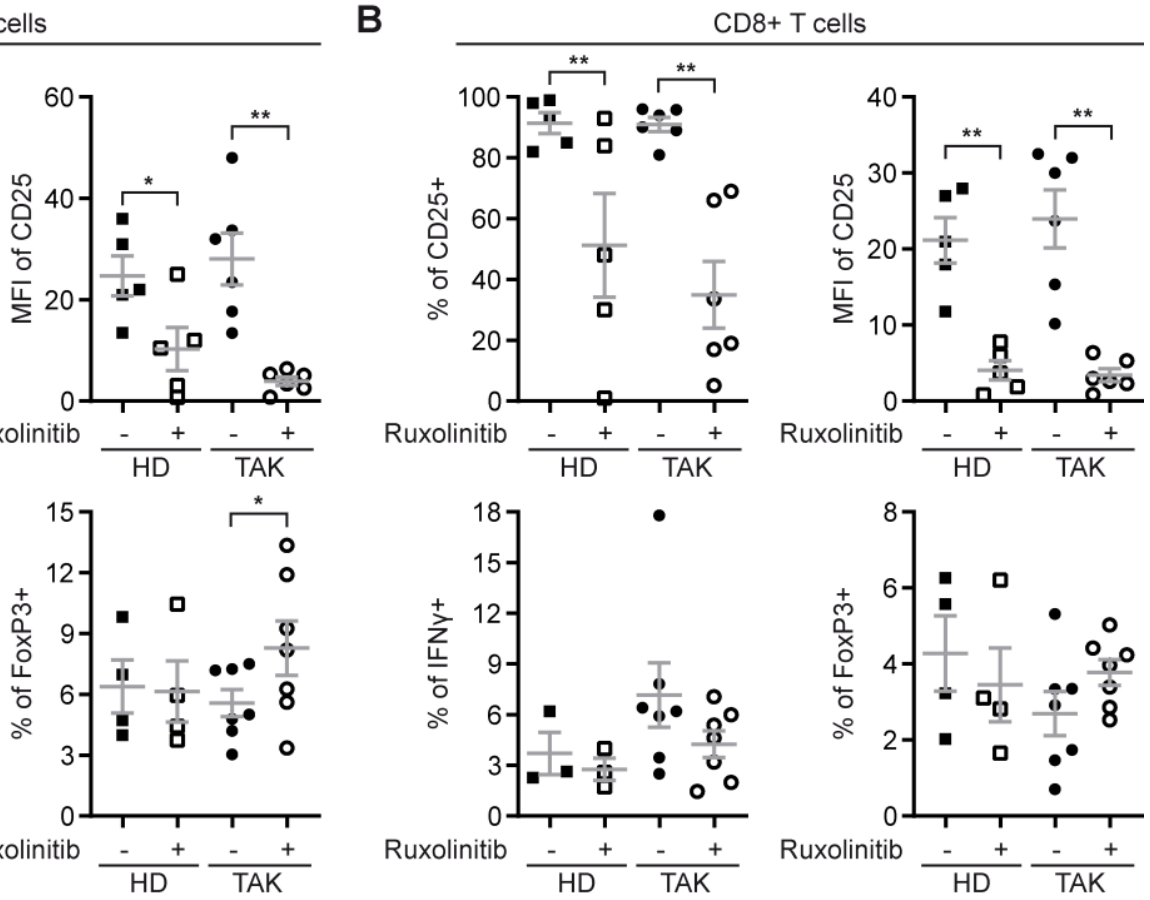
A
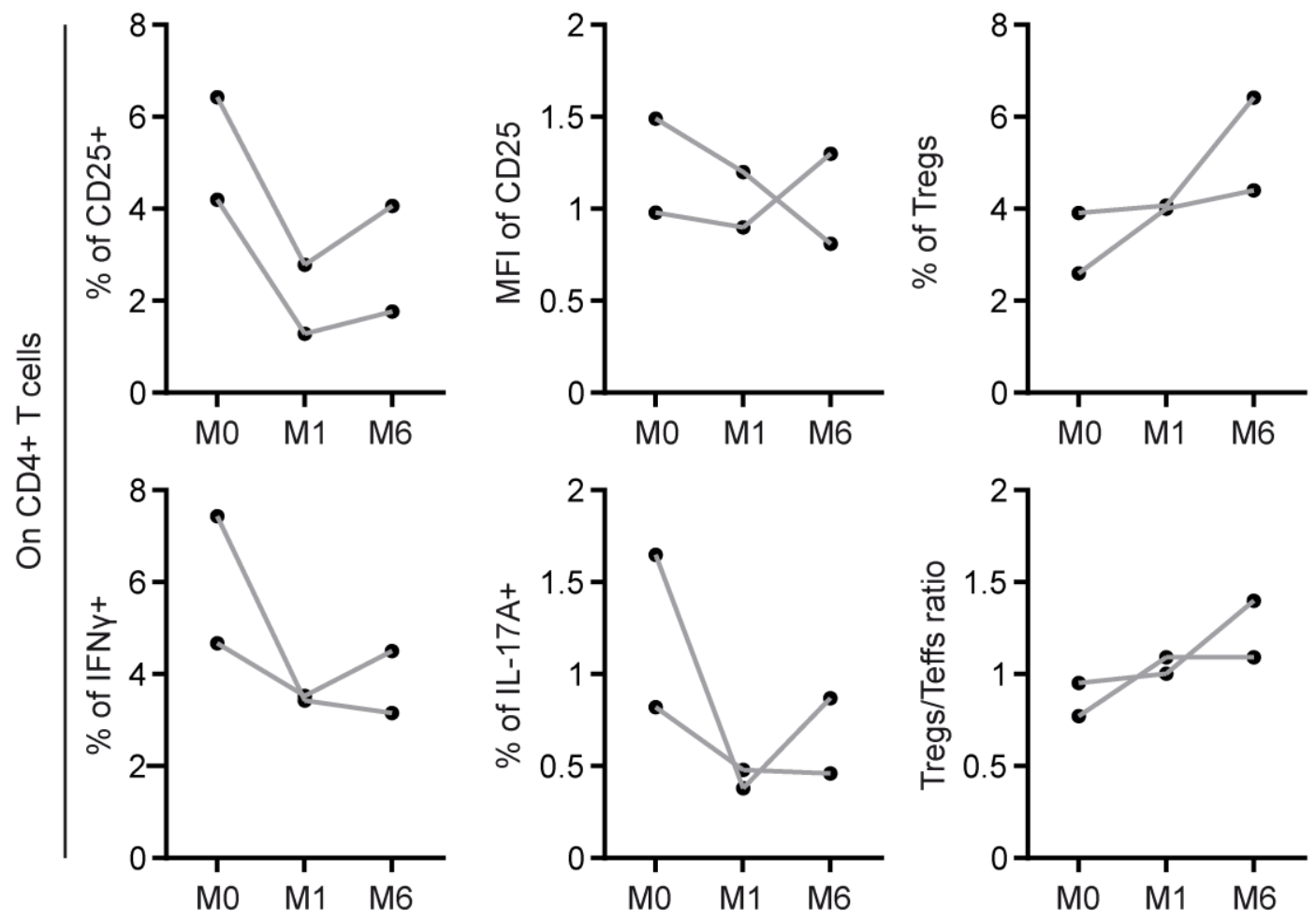

B
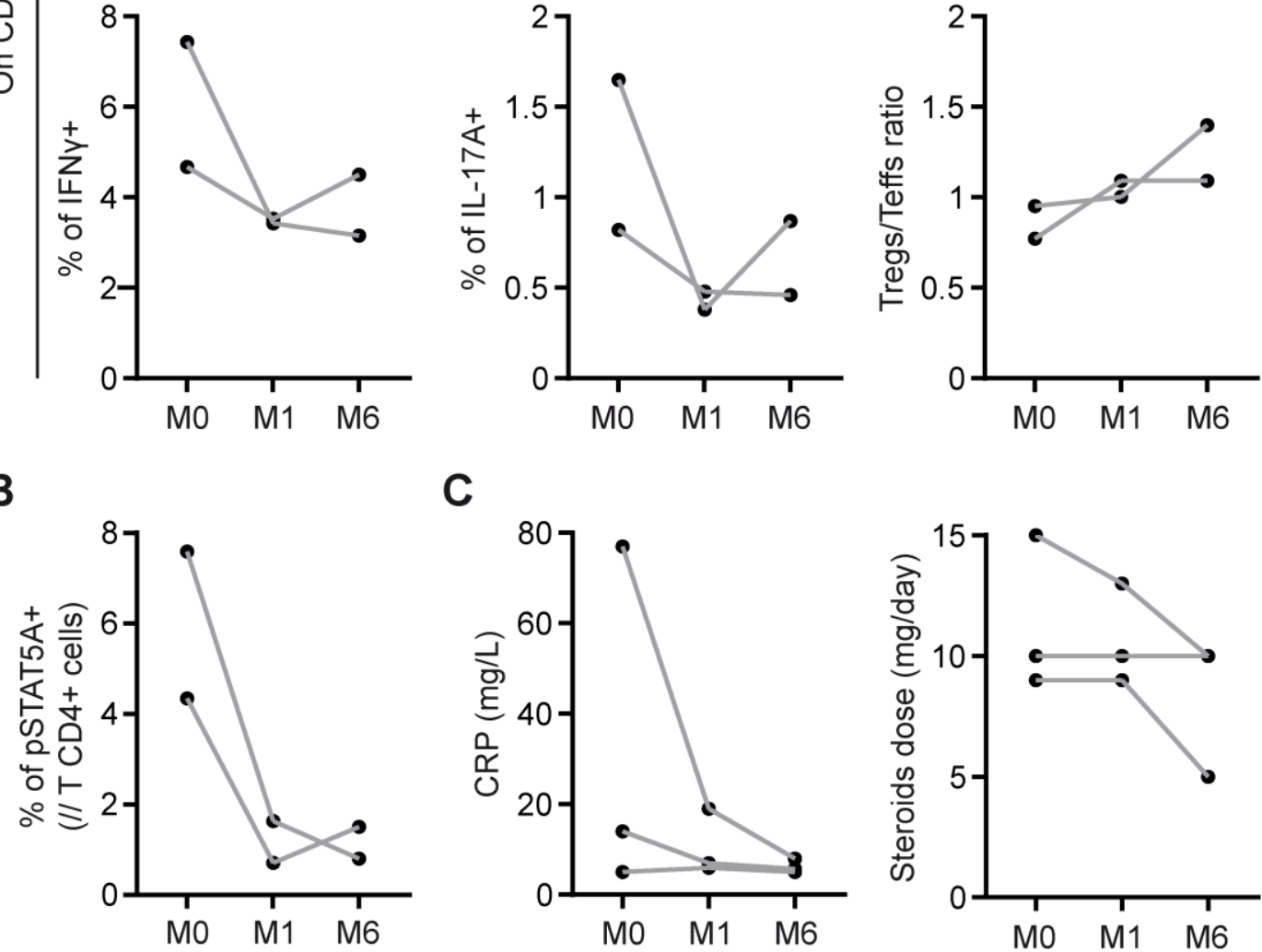

C
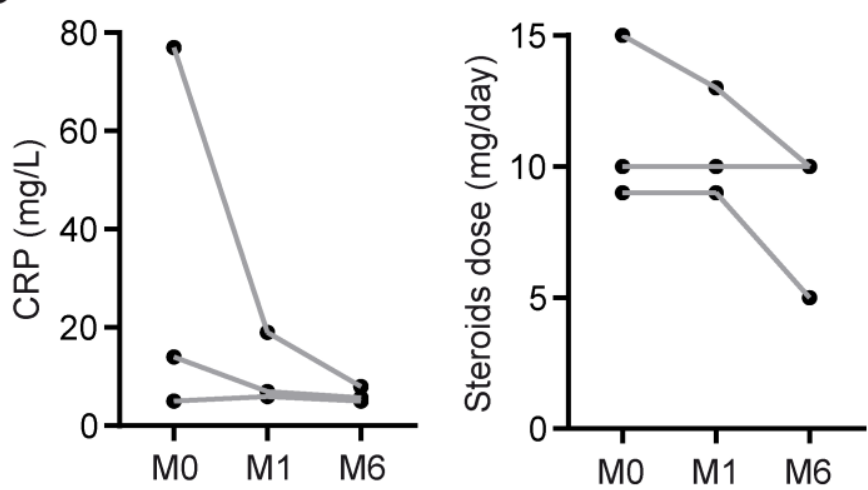

Figure 5 


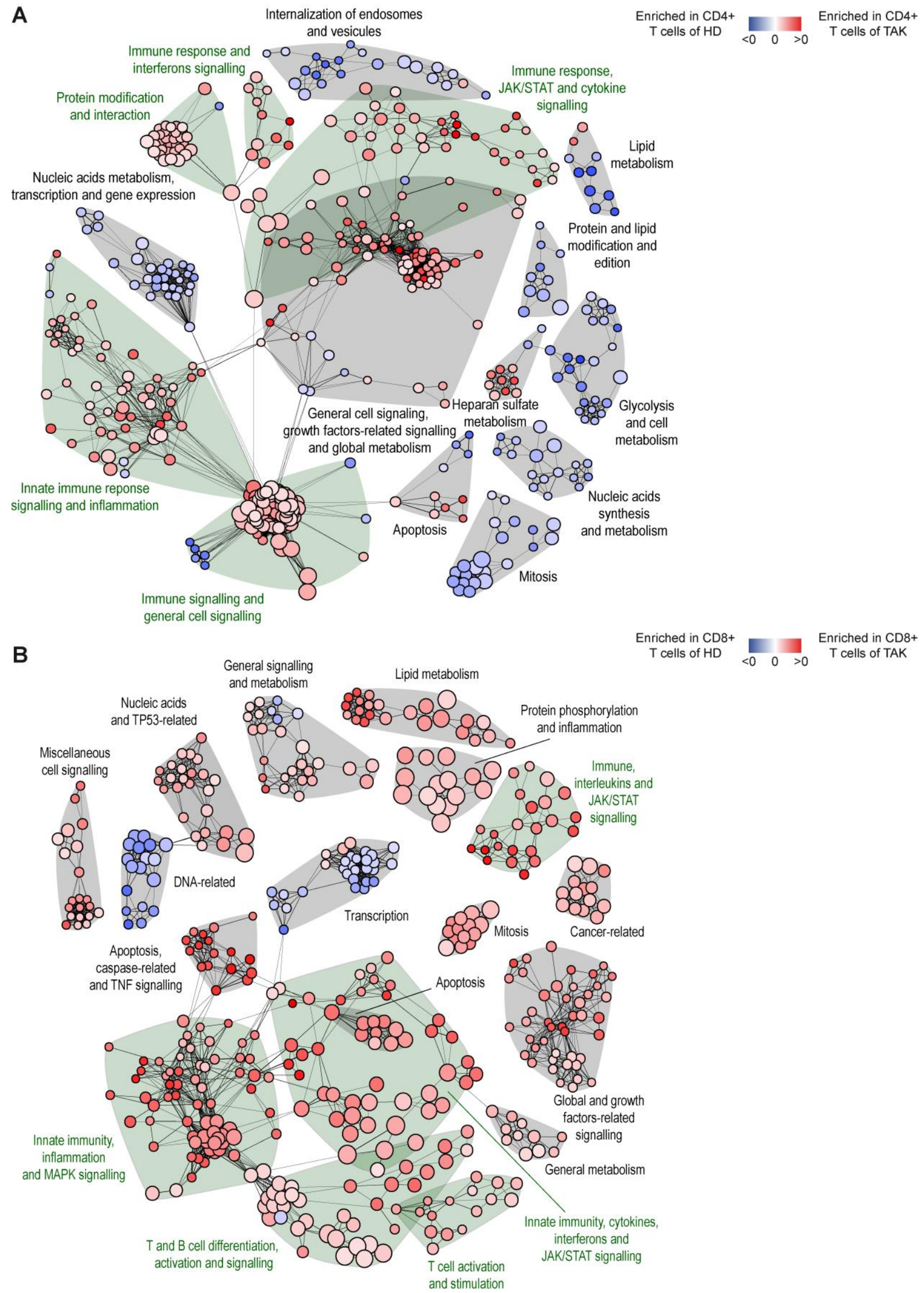

\section{Supplementary Figure 1}


Table 1

\begin{tabular}{lc}
\hline Parameters & TAK (n= 25) \\
\hline Demographic features & \\
Median age [IQR] & $37.6[27.1 ; 50]$ \\
Female gender & $18(72 \%)$ \\
Clinical features & \\
HTA & $14(56 \%)$ \\
Fever & $4(16 \%)$ \\
Asthenia & $12(48 \%)$ \\
Carotydodynia & $11(44 \%)$ \\
Elevated CRP & $13(52 \%)$ \\
Numano Classification & \\
$\quad$ I & $1(4 \%)$ \\
II & $1(4 \%)$ \\
III & $3(12 \%)$ \\
IV & $3(12 \%)$ \\
V & $17(68 \%)$
\end{tabular}




\section{Supplementary Table 1}

\begin{tabular}{|c|c|c|c|}
\hline & P1 & P2 & P3 \\
\hline Age at diagnosis & 50 & 40 & 30 \\
\hline Gender & $\mathrm{F}$ & $\mathrm{F}$ & $\mathrm{M}$ \\
\hline Geographic origin & French West Indies & North Africa (Maghreb) & Caucasian \\
\hline TA features & $\begin{array}{c}\text { Heart, Aortitis } \\
\text { Stenosis: ilio-femoral, } \\
\text { renal, mesenteric, sub- } \\
\text { clavian, carotid, posterior } \\
\text { vertebral arteries } \\
\text { Aneurvsm: none }\end{array}$ & $\begin{array}{c}\text { Stroke, Aortitis } \\
\text { Stenosis: ilio-femoral, } \\
\text { renal, mesenteric arteries } \\
\frac{\text { Aneurvsm: aorta, }}{\text { retinopathy }}\end{array}$ & $\begin{array}{c}\text { Stroke, Aortitis } \\
\text { Stenosis: ilio-femoral, } \\
\text { renal, carotid arteries } \\
\text { Aneurvsm: none }\end{array}$ \\
\hline Numano classification & $\mathrm{V}$ & $\mathrm{V}$ & $\mathrm{V}$ \\
\hline Previous treatment & MMF, TCZ, TNFi, MTX & $\mathrm{MMF}, \mathrm{TCZ}, \mathrm{TNFi}$ & None* \\
\hline $\begin{array}{l}\text { Steroid daily dose at } \\
\text { presentation and at M6 } \\
(\mathrm{mg})\end{array}$ & $10 / 10$ & $9 / 5$ & $15 / 10$ \\
\hline $\begin{array}{l}\text { JAK inhibitor } \\
\text { (type/dose) }\end{array}$ & Baricitinib (4mg/day) & Baricitinib (4mg/day) & Ruxolitinib (10mg/day) \\
\hline $\begin{array}{c}\text { NIH score at } \\
\text { presentation and at M6 }\end{array}$ & $3 / 0$ & $1 / 0$ & $3 / 0$ \\
\hline $\begin{array}{c}\mathrm{CRP}(\mathrm{mg} / \mathrm{dL}) \text { at } \\
\text { presentation and at M6 }\end{array}$ & $77 / 8$ & $5 / 5$ & $14 / 5.7$ \\
\hline
\end{tabular}

